\title{
La cooperación técnica norteamericana en salud pública en Colombia durante la Segunda Guerra Mundial
}

\begin{abstract}
María del Pilar Guzmán¹, Emilio Quevedo²
Resumen

La formulación e implementación de las políticas de salud a nivel local se ve en muchos casos influenciadas por los programas de cooperación que se establecen entre gobiernos o con entidades privadas, los cuales responden a intereses específicos, tanto del país que los ofrece como del país que los recibe. El propósito de este trabajo es analizar los programas de cooperación en salud establecidos entre el gobierno norteamericano y el colombiano entre 1939 y 1945, examinando el significado que tanto para Estados Unidos como para Colombia tuvo la política del interamericanismo, fundamento de muchos de los programas de cooperación en salud. De esta manera, se pretende aclarar la razón por la cual la cooperación en salud se incluye como parte de la agenda en la discusión sobre la seguridad hemisférica, y el impacto de dichos programas en el desarrollo local de las políticas de salud en nuestro país. Se trata de analizar las formas de negociación entre los intereses económicos y políticos involucrados en dicha cooperación y su impacto en el desarrollo de la política de salud pública en Colombia.

Palabras clave: políticas de salud, cooperación norteamericana, programas de cooperación

\section{North American Technical Cooperation in Public Health Policy in Colombia During the Second World War}

\section{Summary}

Formulation and implementation of local health policies is frequently influenced by cooperation programmes established between countries or between private entities having specific interests for the offering country as well as for the receiving country. The purpose of this paper is to analyse the objectives and characteristics of US cooperation in public health with Colombia, between 1939 and 1945. The significance placed by the US (as well as by the Colombian government) on inter-americanism is examined, this being the foundation of many health cooperation programmes. From this standpoint an attempt is made to clarify why health cooperation became part of the agenda in discussions concerning hemispherical security and the impact of such programmes on local health policy development in Colombia. The means of negotiation between economic
\end{abstract}

Este artículo presenta un resumen de los resultados de la Tesis de Maestría en Ciencias Políticas y Relaciones Internacionales realizada por María del Pilar Guzmán y dirigida por Emilio Quevedo, así como parte de los resultados de las investigaciones realizadas en el marco del proyecto El tránsito desde la higiene hacia la medicina tropical y la salud pública en Colombia 18861953.

1 Profesora de la Universidad El Bosque. Investigadora del Instituto Nacional de Salud.

2 Director del Centro de Historia de la Medicina "Andrés Soriano Lleras" de la Universidad Nacional de Colombia. Investigador asociado Instituto Nacional de Salud.

Recibido para publicación 22 de abril de 1998, aprobado 9 de abril de 1999 
and political interests and their effect on the development of public health policies in Colombia is also analysed.

Key words: public health policies, US co-operation, co-operation programmes, hemispherical security, negotiation.

El tema de la cooperación en salud pública comenzó a debatirse en los foros internacionales desde que el aumento de los intercambios comerciales y las facilidades dadas por los transportes en la segunda mitad del siglo XIX pusieron en evidencia la necesidad de tomar medidas de cooperación bilateral por parte de los distintos países para prevenir y combatir enfermedades contagiosas.

En el caso de los países latinoamericanos, el inicio de esta cooperación se materializó con la creación de la Oficina Sanitaria Internacional (posteriormente llamada Oficina Sanitaria Panamericana), en 1903, la cual fue liderada por los Estados Uidos a través de las distintas Conferencias Sanitarias Panamericanas organizadas por esta Oficina; la mayoría de los países latinoamericanos asumieron el compromiso de llevar a cabo actividades de sanidad portuaria y poner en marcha otras reglamentaciones relacionadas con la lucha contra las enfermedades endémicas y epidémicas que pudieran afectar el libre comercio e impidieran una rápida inserción en el mercado internacional.

En Colombia, en las dos primeras décadas de este siglo, dicha cooperación fue brindada por entidades privadas, como la Fundación Rockefeller, a través de programas de erradicación de enfermedades como la uncinariasis (anemia tropical o tun-tun) (2), la malaria y la fiebre amarilla, el apoyo financiero y técnico a institutos y laboratorios de higiene y la formación de personal local en universidades norteamericanas y europeas en las áreas de higiene pública, estadística, ingeniería sanitaria, epidemiología, etc.(1).

La presencia de la Fundación Rockefeller en Colombia, estuvo fuertemente ligada a la expansión de la "economía de enclave" y, especialmente, a la explotación petrolera, pues muchas enfermedades tropicales habían provocado una gran mortalidad en obras como el Canal de Panamá o la construcción de ferrovías y carreteras nacionales (1). Por su parte, el gobierno norteamericano avaló la gestión de estas entidades, ejerciendo su llamada "diplomacia del dólar" con la cual apoyaba las inversiones de sus ciudadanos en el exterior.

Pero sólo fue hasta la década de los treinta cuando, sin dejar de existir la cooperación de instituciones privadas, dicha cooperación se asume como un asunto público manejado de gobierno a gobierno. A partir de ese momento, para los países con mayor desarrollo industrial, la cooperación considerada como un asunto público relacionado con la transferencia o traslado de recursos técnicos, financieros, humanos, etc., a los países de menor desarrollo, adquirió real importancia en el ámbito internacional debido a dos hechos que profundizaron y prolongaron por largo tiempo la desarticulación de los intercambios comerciales y generaron nuevas condiciones geopolíticas: la Gran Depresión y la Segunda Guerra Mundial $(3,4)$.

La coyuntura política provocada por la Segunda Guerra Mundial generó nuevas estrategias de cooperación, todas ellas formuladas en el marco del interamericanismo y de la seguridad hemisférica. Como consecuencia, en este momento se dio un viraje en la política exterior norteamericana respecto a Latinoamérica. La nueva política del buen vecino, puesta en marcha por el presidente Roosevelt, intentó modificar las acciones claramente intervencionistas llevadas a cabo durante las dos primeras décadas de este siglo y planteó relaciones más amistosas con los países de la región (4).

El propósito de este artículo es analizar los programas de cooperación en salud estable-cidos 
entre el gobierno norteamericano y colombiano entre 1939 y 1945, examinando el significado que para Estados Unidos tuvo la política del interamericanismo, fundamento de muchos de los programas de cooperación en salud, al igual que para el gobierno colombiano. De esta manera, se pretende aclarar la razón por la cual la cooperación en salud se incluye como parte de la agenda en la discusión sobre la seguridad hemisférica, y el impacto de dichos programas en el desarrollo local de las políticas de salud en nuestro país.

\section{La buena vecindad y el interamericanismo en tiempos de guerra}

Con la elección de Franklin Delano Roosevelt como presidente de los Estados Unidos en noviembre de 1932, se inició una nueva era en las relaciones con Latinoamérica. Aunque su programa de política exterior se rigió por un marcado aislacionismo, especialmente durante su primera administración, su idea de fortalecer las relaciones interamericanas se desarrolló a través de su famosa política de la "buena vecindad".

Dicho sistema de buena vecindad fue manejado a través del Instituto de Asuntos Interamericanos y se instauró en un momento de tensión en toda la región, debido a las difíciles relaciones con México y Nicaragua y al inconformismo, generado por la discriminación económica implementada en la administración de su antecesor, el presidente Hoover.

Como consecuencia de la Séptima Conferencia Interamericana celebrada en Montevideo en 1933, en la cual se firmó la Convención sobre "Derechos y deberes de los estados americanos", los Estados Unidos tomaron cuatro decisiones políticas que demostraron las intenciones de la nueva administración hacia Latinoamérica, con el fin de buscar acuerdos y soluciones novedosos para los conflictos.

En Cuba (1934), se dejó sin efecto la denominada "Enmienda Platt" que le concedía el derecho a los Estados Unidos de intervenir allí, y fue sustituida por un tratado que dejaba a los norteamericanos únicamente el derecho de ocupar una base naval en Guantánamo.
También en 1934, se terminó la ocupación a Haití.

En 1936, Estados Unidos firmó un acuerdo con Panamá, que la convertía en un protectorado de los E.E.U.U. y estipulaba la defensa conjunta del Canal.

En México, se buscó un acercamiento al problema de la nacionalización de los campos petroleros, creando una comisión conjunta para evaluar las propiedades y determinar el valor compensatorio a los inversionistas norteamericanos (5).

Un símbolo adicional del cambio en la política externa de los Estados Unidos con respecto a Latinoamérica, fue el reconocimiento de la soberanía nacional de Nicaragua y Honduras, al someter el litigio de fronteras a un arbitramento de una tercera nación latinoamericana.

La preocupación por el mejoramiento de las condiciones económicas y sociales como parte de la estabilidad política de los países latinoamericanos estuvo presente en las propuestas de cooperación del gobierno norteamericano en las distintas reuniones panamericanas. Así lo manifestaba Roosevelt en una conferencia de prensa de 1941: "los mejores intereses de los Estados Unidos están en cooperar con los países de América Latina y ayudarlos a aumentar su productividad, sus niveles de vida y su poder de compra" (5).

Después de la Tercera Conferencia de Ministros de Relaciones Exteriores de las Repúblicas Americanas, se estableció el programa cooperativo entre el Instituto de Asuntos Interamericanos, entidad dependiente del gobierno de Washington y las 21 repúblicas americanas, para implementar un plan de salubridad y saneamiento.

\section{La cooperación técnica en el campo de la salud en Colombia como parte de la política del buen vecino}

En ese marco, se logró concretar un programa de cooperación entre la División de Saneamiento y Salud Pública del Instituto de Asuntos Interamericanos y el gobierno colombiano, como un mecanismo para fortalecer la solidaridad hemisférica y reafirmar la buena vecindad. 
El gobierno colombiano solicitó entonces las bases para participar en este programa, e inició las gestiones necesarias para que Colombia pudiera beneficiarse. Para ello, preparó un memorándum sobre las posibles actividades cooperativas de salubridad en Colombia el cual se entregó a Nelson Rockefeller, coordinador de la Oficina de Relaciones Interamericanas.

Nelson Rockefeller realizó un viaje a Suramérica en septiembre de 1942 con el fin de estudiar los problemas creados por la guerra y establecer programas de cooperación en diferentes áreas. A Bogotá llegó el 23 de septiembre de 1942, y el Ministro de Trabajo, Higiene y Previsión Social, Arcesio Londoño Palacio, le presentó los siguientes problemas como posibles áreas de cooperación:

1. Nutrición: para lo cual se propuso la creación de un instituto que estudiase las necesidades fisiológicas de los colombianos, la composición química y las condiciones higiénicas de los alimentos en el país, el estudio de las enfermedades de origen alimenticio y la formación de personal capacitado de médicos, visitadores sociales y enfermeras, expertos en cuestiones de alimentación.

Además, se planteó la implementación de restaurantes y huertas escolares, conferencias a la comunidad sobre hábitos de alimentación y orientación en cuanto a su presupuesto, divulgadas también a través de la radio y la prensa.

2. Paludismo o malaria: enfermedad muy sentida entre la población colombiana, especialmente en las tierras bajas (zonas arroceras) y en la cuenca de los ríos, la cual provocaba 18.000 víctimas anualmente. Para elio, se sugirió la formación de malariólogos especializados, el saneamiento en los grandes ríos y la intensificación del cultivo de quina.

3. Rickettsiasis o tifo exántemático: que requería principalmente educación en aseo personal, destrucción de parásitos y roedores, y vacunación intensiva.

4. Bartonelosis: enfermedad a la cual se pedía estudiarla con detenimiento para conocer los mecanismos de transmisión y los métodos terapéuticos, ya que no se disponía de tratamientos específicos.

5. Saneamiento de puertos: a través de la construcción de acueductos, alcantarillados y obras sanitarias en los principales puertos del país (Barranquilla, Cartagena, Santa Marta, Buenaventura, Tumaco y Bahía Solano).

De esta reunión, salió entonces el convenio celebrado entre los gobiernos de Colombia y Estados Unidos para la ejecución del nuevo programa de salubridad y saneamiento (6).

El director de la División de Salubridad y Saneamiento de la oficina del coordinador de relaciones Interamericanas, el brigadier general George C. Dunham escribió una carta al Ministro de Trabajo, Higiene y Previsión Social para informarle acerca de los requisitos que habrían de emplearse en la ejecución del programa cooperativo; dichos requisitos se pueden resumir así:

1. La creación de un servicio técnico especial que se conocería como el Servicio Cooperativo Interamericano de Salud Pública (SCISP), y que serviría como intermediario para la cooperación entre el gobierno de Colombia y el Instituto de Relaciones Interamericanas, siendo una entidad separada pero dependiente directamente del Ministerio de Trabajo, Higiene y Previsión Social.

2. La creación de una misión pagada por el Instituto de Relaciones Interamericanas que trabajaría en Colombia y cuyo jefe sería el director del SCISP, quien, además, seleccionaría el personal y las condiciones de empleo de sus colaboradores, acordadas previamente con el Ministro ${ }^{4}$.

3. Los fondos para la financiación del S.C.I.S.P. se obtendrían mediante "la contribución de una suma, no mayor de un millón de dólares americanos, por parte del Instituto de Relaciones Interamericanas y de una suma, no mayor de un millón de pesos colombianos, por parte del gobierno colombiano".

Sin embargo, esta proporción sería modificada rápidamente, pues, para 1946, por cada dólar 
asignado por los E.E.U.U., el gobierno colombiano aportaba 10 dólares en moneda corriente, al cambio oficial. La idea era que el gobierno colombiano fuera asumiendo progresivamente los programas ya montados por el S.C.I.P.S.

Cualquier obra específica que ejecutara el S.C.I.S.P. sería motivo de convenio o contrato separado, o de ambos a la vez, entre el Ministro y el representante del Instituto de Relaciones Interamericanas en Colombia, al igual que si se hiciera con otra entidad jurídica o individual.

Las autorizaciones de desembolso de los fondos del S.C.I.S.P. se harían conjuntamente.

El director del S.C.I.S.P. tendría que rendir informes mensuales acerca de sus obras específicas y anuales de la contabilidad de todos los compromisos, transacciones y desembolsos.

Finalmente, se propuso que el S.C.I.S.P. interviniera en el saneamiento de puertos, el dominio de las enfermedades endémicas, el establecimiento de centros de higiene, el mejoramiento de la nutrición y la preparación de personal colombiano en salubridad, ingeniería sanitaria, enfermería, administración de hospitales y otros ramos similares (7).

De esta manera, el S.C.I.S.P. fue autorizado por la Ley 36 de 1942 y mediante el Decreto número 41 de 1943 se creó como un organismo dependiente del Ministerio de Trabajo, Higiene y Previsión Social.

Los objetivos de este programa cooperativo de salud y saneamiento, como se expresa en la cláusula primera del acuerdo básico eran:

1. Promover y fortalecer la amistad y el entendimiento entre los pueblos de la República de Colombia y los Estados Unidos y fomentar su bienestar general.

2. Asistir en las actividades de salud pública y saneamiento en Colombia por medio de la acción y cooperación de las partes contratantes de este convenio.

\footnotetext{
4 Valga la pena comentar que el modelo utilizado es muy similar al del Departamento de Uncinariasis, organizado por la Rockefeller en la primera mitad del siglo XX (2).
}

3. Estimular y acrecentar entre los países el intercambio de conocimientos, pericias y sistemas técnicos en el campo de la salud pública y el saneamiento (7).

EI S.C.I.S.P. se convirtió entonces en un organismo regional (creado en casi todos los países latinoamericanos), subsidiario del Instituto de Relaciones Interamericanas, de carácter burocrático, constituido como una organización formal, con reglas establecidas, con registros de sus propias operaciones y con una jerarquía definida en los diversos niveles.

\section{La verdadera agenda del programa de cooperación técnica en salud pública en el marco del interamericanismo}

Lo que realmente pretendía la política del interamericanismo era extender el neutralismo a todo el continente americano en caso de guerra. Para ello se planteó la tesis de que "la amenaza a un estado americano, significaría la amenaza a la seguridad de todos los demás países de América" (8).

Las acciones puestas en marcha crearon un ambiente favorable a las relaciones interamericanas, y posibilitaron los acuerdos posteriores en torno al programa norteamericano del "mantenimiento de la paz" y la aplicación de medidas "panamericanas" conjuntas para el enfrentamiento con los países del eje durante la Segunda Guerra Mundial.

Según Duroselle, dicha tesis "no era algo puramente académico, ya que por la considerable inmigración alemana e italiana hacia Argentina y Brasil, existía una verdadera red de subversión nazi y muchos norteamericanos desconfiaban del papel que podría desempeñar en un momento dado la compañía aérea alemana Lufthansa, que había logrado ocupar una gran parte del tráfico latinoamericano" (9).

Esta tesis no fue acogida en su totalidad por los países latinoamericanos, pero se logró crear un comité panamericano permanente de consultas compuesto por ministros de relaciones exteriores de los países americanos.

Las leyes de neutralidad de 1935 y 1936 fueron aprobadas por el congreso norteamericano, ante 
el anuncio del rearme alemán (17 de marzo de 1935), el comienzo de la guerra de Etiopía (3 de octubre de 1935), la remilitarización de Renania (7 de marzo de 1936), y el inicio de la guerra civil española (julio de 1936); por ello, la posición adoptada por el gobierno de Roosevelt fue claramente aislacionista.

Esta política se justificó fundamentalmente por dos aspectos: primero, ante la crisis económica mundial, Roosevelt estaba convencido de que ésta debía resolverse internamente, de ahí su programa de New Deal (construcción de obras públicas, subsidios al desempleo, ayuda agrícola, etc.) y segundo, como a los norteamericanos les había tocado asumir la deuda de la Primera Guerra Mundial de los europeos, la opinión pública no estaba de acuerdo con intervenir en problemas relacionados con el viejo continente.

La extensión de la política de neutralidad a América Latina vino a convertirse, en una forma de "vinculación entre la política interamericana y los acontecimientos europeos"; de esta manera, el panamericanismo fue utilizado como un foro mundial, en el que Roosevelt globalizó las relaciones interamericanas, ubicándolas como las relaciones de Estados Unidos con el resto del mundo (5).

En la primera reunión de consulta de Ministros de Relaciones Exteriores (Panamá, 1939), se acordó la defensa de un perímetro alrededor de las Américas, desde Terranova hasta la Tierra del Fuego, y un parámetro de doscientas millas en todas las áreas costeras, áreas en las cuales no se permitiría el paso de los beligerantes. Esta zona de seguridad fue ampliada unilateralmente por los Estados Unidos en abril de 1941 a Groenlandia, mediante un convenio con el gobierno danés. Igualmente, con la invasión alemana a Rusia se enviaron tropas a Islandia con la justificación de la "defensa hemisférica", aunque lo que realmente se buscaba era proteger las rutas del comercio de los E.E.U.U. hacia la Gran Bretaña.

En la segunda reunión de consulta de Ministros de Relaciones Exteriores (La Habana, 1940) se adoptó una política de administración provisional colectiva de las colonias de los estados europeos en América, como instrumento de presión contra
Alemania y los países del eje durante la guerra. Sin embargo, nunca se llevó a la práctica.

Además, en ese mismo año las repúblicas americanas firmaron una "Declaración de asistencia recíproca y cooperación para la defensa de las naciones de América" que afirmó que cualquier interferencia con la soberanía de una nación americana sería considerada como un ataque a todas ellas. "Este fue el primer acuerdo interamericano de seguridad, dirigido a los países no americanos, que vino a ser el fundamento de varios convenios en tiempos de guerra" (5). El objetivo de los Estados Unidos era crear bajo su égida un tercer bloque de países "neutrales".

Con la entrada definitiva de Estados Unidos a la guerra, por la invasión japonesa a Pearl Harbor el 7 de diciembre de 1941, los E.E.U.U. empezaron a promover en el marco del multilateralismo panamericano ya no una política de neutralidad, sino de defensa contra la agresión de los países del eje.

De esta manera, en la mencionada Tercera Reunión de Ministros de Relaciones Exteriores, realizada en Río de Janeiro en enero de 1942, se recomendó que los países americanos rompieran relaciones diplomáticas, comerciales y financieras con Japón, Italia y Alemania, y además, se creó un consejo interamericano de defensa, encabezado por un general norteamericano, con el objeto de controlar la actividad "subversiva nacista" y la coordinación de medidas judiciales y policiales.

\section{Las relaciones Colombia-Estados Unidos y el panamericanismo desde la perspectiva colombiana}

La Segunda Guerra Mundial consolidó la tendencia que existía desde los gobiernos de Marco Fidel Suárez (1918-1921) y Enrique Olaya Herrera (1930-1934) de mantener relaciones estrechas con la "Estrella Polar" ya que, según sus planteamientos, los vínculos con este país eran inevitables, y las relaciones debían manejarse de manera constructiva. Eduardo Santos, quien había sido elegido como presidente liberal en 1938, fomentó la cooperación con los norteamericanos en el área económica, política y militar, en momentos en que las relaciones 
internacionales globales se deterioraban y la seguridad y solidaridad hemisféricas se hacían muy urgentes.

Además, nacionalizó la aerolínea Scadta, controlada por los alemanes, colocando la empresa bajo la administración colomboamericana (Avianca-Pan American Airways), y rompió relaciones con los países del eje poco después del ataque a Pearl Harbor (1941).

Igualmente, se acordaron medidas conjuntas defensivas como: entrenamiento de personal militar, préstamos de asesores militares, intercambio de información relativa a la seguridad, patrullajes costeros y al desarrollo de un sistema de contraespionaje a través de la embajada y los consulados americanos (4).

Los vínculos comerciales entre Latinoamérica y los E.E.U.U. también se estrecharon, pues la guerra transformó los canales de comercio y transporte internacional. En el caso colombiano, por ejemplo, en 1934 , el $84 \%$ de las exportaciones de café se hacía a Alemania, mientras que, en 1938 , el $80 \%$ se hacía a los Estados Unidos; y el suministro de maquinaria y equipo para el país y de algunos bienes como hierro y llantas, se complicó de manera dramática, teniendo que recurrir al mercado norteamericano, el cual tampoco pudo responder a estas demandas especialmente cuando E.E.U.U. ingresó a la guerra en 1941 (10).

En este contexto, asumió por segunda vez la presidencia Alfonso López Pumarejo (1942), quien auspició y respaldó la "buena vecindad" (11). López apoyó la propuesta de la Tercera Conferencia de Ministros de Relaciones Exteriores realizada en Río de Janeiro en 1942 con respecto a la venta exclusiva a E.E.U.U. de recursos estratégicos.

En este sentido, los Estados Unidos diseñaron un programa de compra de toda la producción colombiana de caucho, carbón y platino, exigiendo fuertes controles para evitar el contrabando de estos productos a los países del eje, pues se tenía el dato de que, en 1938, Alemania había aumentado enormemente las compras de platino colombiano (3).
Dicho programa fue fundamental para los Estados Unidos, pues, entre 1943 y 1944, estaban construyendo un barco diario y un avión cada cinco minutos. Por ello, el presidente Roosevelt calificó a su país de "arsenal de las democracias" y en opinión de David Thompson, en los seis años de guerra, ese arsenal produjo 87.000 tanques, 296.000 aviones, 350.000 piezas de artillería y morteros, 2'434.000 camiones y $53^{\prime} 000.000$ de toneladas marítimas (12).

A cambio de la ayuda que prestó Colombia para poder construir un sistema de seguridad hemisférica, los Estados Unidos aumentaron la ayuda económica a través de préstamos. Según Stephen Randall, por ejemplo, "el Export Import Bank concedió tres préstamos substanciales a Colombia durante la guerra: 20 millones de dólares en 1941 para la construcción de carreteras; 10 millones de dólares en 1943 para proyectos agrícolas y 3,4 millones de dólares en 1944 para la construcción de una planta hidroeléctrica" (3).

Los préstamos para transporte fueron acogidos con gran agrado por la industria cafetera, ya que para la comercialización del café, el río Magdalena y los ferrocarriles que trasladaban el producto hasta Buenaventura resultaban insuficientes por la congestión que el volumen ocasionaba. Por ello, el gremio cafetero "veía con simpatía el estrechamiento de las relaciones entre Colombia y los Estados Unidos" (13).

Esteban Jaramillo, presidente de la Federación de Cafeteros, en 1940 expresaba al respecto: "Dependemos económicamente, hoy más que nunca de los Estados Unidos (...) y terminado el conflicto bélico, es probable que aquella vinculación sea aún más fuerte si se realiza el pensamiento de una gran confederación económica americana, que regule y dirija la economía y las finanzas de los países de este hemisferio entre sí" (14).

Además, la coyuntura de la guerra y el deseo de Santos y también de López de adherirse a los principios de solidaridad hemisférica, facilitaron la modificación de la legislación petrolera colombiana en beneficio de los Estados Unidos. 
En la industria del petróleo, los principales obstáculos que encontraban los inversionistas extranjeros tenía que ver con la legislación de 1936, lo cual planteó entre otras cosas:

1. La imposibilidad de que una sola persona o entidad obtuviera derechos de exploración o explotación por más de 100.000 hectáreas, con excepción de los Llanos Orientales, donde el máximo eran 200.000. Este aspecto de la legislación fue evadido, según Bushnell, a través de la constitución de filiales o terceras personas, para obtener concesiones adicionales (4).

2. La obligación de que las compañías iniciaran perforaciones antes de finalizar el período asignado para la explotación inicial, para evitar que éstas se dedicaran a acumular concesiones. Durante la guerra, se suspendieron los requisitos de perforación por la escasez de acero y de equipos de perforación.

3. El deber de las empresas extranjeras de mantener un porcentaje mínimo de nacionales entre sus empleados, y que su remuneración fuera igual a la de sus trabajadores. EI problema para el cumplimento de esta ley era que no existían suficientes profesionales colombianos en el campo; por ello, su aplicación fue muy flexible.

De esta manera, en el contexto de la guerra, los E.E.U.U. lograron ganar no sólo aliados políticos, sino socios económicos en el país. Desde la perspectiva latinoamericana, la coyuntura permitió estrechar más las relaciones con el vecino del norte, ya que era el centro de sus intercambios comerciales, y el país que se vislumbraba como hegemónico en el sistema internacional de la posguerra.

Por ello, la política exterior colombiana en ese momento se orientó a la causa aliada y adoptó todos los acuerdos interamericanos de defensa continental.

\section{La salud pública: ¿una cuestión de defensa hemisférica?}

En el marco de la política del buen vecino y en el desarrollo de la Tercera Reunión de Consulta de Ministros de Relaciones Exteriores de las repúblicas americanas realizada en Río de
Janeiro del 15 ai 28 de enero de 1942, se planteó como una prioridad la protección del hemisferio occidental, y el estudio de medidas para restablecer el orden mundial, alterado desde 1939 por la Segunda Guerra Mundial.

Para lograr este objetivo, se trataron temas como la solidaridad económica, la ruptura de relaciones diplomáticas con los países del eje, la producción de materiales estratégicos, la movilización de medios de transporte, el desarrollo del intercambio comercial, la condenación de la agresión japonesa, la aviación civil y comercial, y también el mejoramiento de la salud y de la salubridad pública.

Así, la cooperación en salud quedó planteada en la resolución $X X X$, considerando, por una parte, que la defensa del hemisferio occidental requería la movilización de las fuerzas vitales, humanas y materiales de las repúblicas americanas $y$, por otra, que tomar medidas adecuadas para proteger la salud y mejorar la salubridad pública constituía un aporte esencial al mantenimiento del poder de los pueblos de América para defenderse y resistir la agresión.

Por esas razones, esta Tercera Reunión de Consulta recomendó a los gobiernos de las repúblicas americanas que, individualmente 0 mediante acuerdos complementarios entre dos o más de ellas, tomasen las medidas necesarias para solucionar los problemas de salubridad e higiene, contribuyendo según su capacidad con materias primas, servicios y fondos.

Igualmente, recomendó que, para la realización de estos objetivos, fuesen utilizadas la ayuda técnica y el consejo de los servicios nacionales de sanidad de cada país en cooperación con la Oficina Sanitaria Panamericana (15).

Así, se puede deducir claramente de esta resolución que la cooperación en salud pública no tenía en ese momento como objetivo fundamental la solución de problemas de la región latinoamericana, los cuales eran muchos, sino apoyar a la política de defensa del hemisferio contra una posible intervención de los países del eje. Incluso, en las consideraciones expuestas en dicha resolución, se plantea que los planes y 
objetivos comunes en esta área "han de contribuir a la reconstrucción del orden mundial" (15).

De igual forma, en la Undécima Conferencia Sanitaria Panamericana, celebrada también en Río de Janeiro, en septiembre de 1942, se planteó que ante el peligro de que se extendieran más las hostilidades en el hemisferio occidental, se hacía necesario intensificar los preparativos de la defensa en el campo de la sanidad pública, fomentando el intercambio de recursos para la conservación de esta área, racionalizando dichos recursos y adoptando medidas extraordinarias para prevenir la diseminación de las enfermedades, ante el incremento de los transportes aéreos, tanto civiles como militares. Además de lo anterior, se pidió que se incluyeran cursos de especialización en higiene y salud pública, en la cátedra de medicina militar (16).

También en las revistas especializadas en medicina, se concebía la cooperación interamericana en salud, en términos de la defensa hemisférica. Por ejemplo, en la Revista de Higiene se decía, en 1944, que "una América unida en sus aspiraciones e ideales para el cuidado de los enfermos y heridos, sería lo eminentemente deseable en estos tiempos, cuando a los problemas de la preservación de la salud de la población civil, se añaden aquellos de la preparación médica para la defensa del hemisferio" (17).

Así, pues, la solidaridad y cooperación técnica en asuntos como la salud pública y el mejoramiento de los niveles de vida y la capacidad de producción, en el marco de la idea de la "comunidad panamericana", tuvieron como fundamento la seguridad colectiva en tiempos de guerra.

\section{Impacto de los programas de cooperación en el desarrollo local de las políticas de salud pública}

La presencia de una entidad como el SCISP, cuya existencia se va a prolongar hasta la década de los sesenta, hace necesario evaluar ciertos aspectos como: la priorización de sus programas, su grado de coordinación con otras secciones del Ministerio encargado de la salud, su impacto en la formación del recurso humano y en la reducción de la morbilidad y mortalidad. El exa- men de estos elementos permite comprender el ajuste de dichos programas a las condiciones y necesidades del desarrollo nacional y su injerencia en la formulación e implementación de las políticas de salud pública nacionales.

1. La priorización de los programas: analizando los proyectos que el SCISP emprendió tales como: sanidad en los puertos, creación de centros de higiene, control del paludismo en las zonas de cultivo de arroz, en la zona bananera y en las explotaciones de caucho, las labores en el área de nutrición como la instalación de restaurantes populares y las campañas contra el coto simple (enfermedad provocada por la carencia de yodo en la sal de consumo y la mala calidad de las aguas), la construcción de letrinas y los proyectos posteriores a 1945 como la campaña antipiánica, la campaña contra la malaria y el control de insectos, la educación sanitaria, el entrenamiento de personal, la higiene industrial, la ingeniería sanitaria, etc., en todos ellos, predomina la idea de que "asegurar salud es posibilitar producción y lograr producción es mejorar salud".

Esta afirmación se corrobora en las siguientes citas, las cuales corresponden a los informes de los proyectos anteriormente nombrados:

- "Si se llevara a cabo la erradicación de la enfermedad se incorporarían a la actividad económica cerca de 250.000 personas, cuyo aporte laboral se pude apreciar en $\$ 300.000$ al año, por lo menos" (18).

- "La malaria económicamente afecta al país, con una pérdida anual de 64 millones de pesos, por concepto de defunciones, incapacidad y cesantías de trabajo, de cerca de 360.000 personas en edad activa (19).

Incluso posteriormente, en el año de 1950 cuando se contrata la Misión Currie, con el objeto de estudiar las posibilidades y problemas de desarrollo del país, se argumenta que: "los bajos niveles de salud causan muchos costos directos e indirectos, y desvían fondos y facilidades que podrían ser empleados para incrementar y fortalecer la economía. La salud deficiente es costosa, en términos de tiempo perdido en la 
finca o en el lugar de trabajo, y desde el punto de vista de las enormes necesidades que crea como hospitales e instalaciones de servicio médico, e igualmente por el aumento de los costos de beneficencia, seguros, indemnizaciones por enfermedad y accidentes" (20).

Con ello, se observa la forma como se impone el análisis economicista en las decisiones políticas en salud, al punto de asumir el criterio de bienestar económico como el más adecuado, dejando de lado el propósito de la formulación e implementación de una política social que responda de manera más efectiva a las necesidades de la población y cuya evaluación no sea medida únicamente en términos económicos de costo-beneficio.

b. Impacto en las instituciones de salud: Las sugerencias dadas por el SCISP en materia de creación de instituciones o secciones especiales dentro del ministerio, fueron en su mayoría, tenidas en cuenta por el gobierno colombiano. Tal es el caso de la creación dentro del Instituto Epidemiológico Nacional (inaugurado el 2 de diciembre de 1942) de un Laboratorio de Nutrición para investigar todo lo pertinente a los alimentos nacionales, y de un Instituto Nacional de Nutrición (1947), el Departamento de Malariología (1946), la División de Bioestadística y la construcción de un edificio para la Escuela Superior de Enfermeras, en la Ciudad Universitaria. (El Instituto de Asuntos Interamericanos, contribuyó con la mitad de los gastos del nuevo edificio y pagó los sueldos de la Directora y de la Directora Asociada).

Igualmente, el SCISP mantuvo estrechas relaciones con entidades como el Instituto de Estudios Especiales "Carlos Finlay", debido a que la Fundación Rockefeller, financiaba en parte, tanto las actividades del Instituto, como las de muchos de los proyectos de educación sanitaria, entrenamiento de personal, campaña de malariología y campaña de control de insectos formuladas por SCISP. También, mantuvo estrechas relaciones con otras dependencias del Ministerio de Higiene como el Instituto Nacional de Higiene Samper-Martínez, el cual le facilitó el uso del serpentario de Armero en 1943 con el fin de que ese estableciera en esa dependencia un centro de investigación del paludismo y le colaboró con la realización de exámenes de laboratorio.

Sin embargo, la falta de articulación de las diferentes secciones del ministerio y de centralización de sus actividades era una queja permanente entre los directores de sanidad del país, así lo confirma la siguiente afirmación de Jorge Bejarano, quien iría a ser luego el primer Ministro de Salud del país: "es un hecho aberrante que la persona o entidad que contribuye económicamente en actividades de salubridad, se cree con el derecho de compartir la atribución técnica y administrativa, derecho que debe ser exclusivo de la directiva del ramo, porque se forma el falso criterio de que cooperación es descentralización" (21).

Para 1945, el balance que Bejarano hacía de la cobertura y calidad de los Centros de Higiene del SCISP, y también de todos los instalados por el gobierno era defectuoso, pues según él: "la generalidad de las poblaciones continúa contemplando sus mismos problemas sanitarios de hace remotos años "ni el acarreo de basuras, ni el conveniente depósito de ellas en los domicilios, ni incineraciones, ni agua potable para la ciudad, ni matadero higiénico, ni higiene de la leche y demás alimentos, ni lucha contra la mosca doméstica, el mosquito y otros vectores de enfermedades" (21).

Parte de la ineficiencia de estos Centros y de los problemas de la organización sanitaria en Colombia se atribuían a:

La falta de un plan centralista que propusiera una unidad de criterio y de liderazgo en la labor de salubridad.

La carencia de una cátedra de higiene que sirviera para formar teórica y prácticamente al personal.

La necesidad de mayor autonomía del Departamento Nacional de Salubridad con respecto al Ministerio de Trabajo. Esto último se logró mejorar cuando a finales de 1946 cuando se creó el Ministerio de Higiene, con una organización independiente del Ministerio de Trabajo. 
c. Impacto en la formación del recursos humano en salud: El apoyo brindado tanto por el SCISP como por la Fundación Rockefeller en la formación de personal sanitario en el exterior se dio en áreas como la Salud Pública, la Ingeniería Sanitaria, Enfermería, Higiene Industrial etc. y, la asistencia técnica de consultores norteamericanos especializados en los programas de saneamiento de puertos, en educación sanitaria y en enfermedades tropicales como la fiebre amarilla y el paludismo. Los cursos que financiaba el Proyecto No. 502 destinado específicamente al Entrenamiento de Personal eran de dos tipos:

- Cursos menores de un año (generalmente 30 4 meses) y viajes de observación al exterior.

- Cursos académicos de un año de duración, en distintas ramas de la Salud Pública.

Entre 1944 y 1952, el Instituto de Asuntos Interamericanos a través del SCISP concedió 300 becas, la mayor parte de los becados trabajaban en labores de docencia y estaban vinculados a instituciones gubernamentales. Por su parte, la Fundación Rockefeller amplio los cupos de personal latinoamericano durante la Segunda Guerra Mundial debido a que esta coyuntura provocó la disminución de becas para estudiantes europeos. Según el Reporte Anual de dicha Fundación: "en 1939 se concedieron becas a 151 estudiantes americanos, 141 europeos y 47 latinoamericanos, mientras que en 1943, la proporción fue de 58 norteamericanos, 4 europeos y 107 latinoamericanos" (22)

La cooperación prestada por el SCISP en cuanto a formación de profesionales y funcionarios públicos en el extranjero tuvo entonces, como incidencia a nivel nacional, por un lado, el afianzamiento del modelo de Salud Pública norteamericano, y por el otro, la proliferación paulatina de especialidades médicas. Este último aspecto, es más notorio con la adopción de las recomendaciones de las misiones médicas norteamericanas Humpherys (1948) y Lapham (1953), las cuales introdujeron el modelo flexneriano en el curriculum de las principales facultades de medicina del país y cambios en las instituciones hospitalarias.

d. Impacto en la reducción de la morbi-mortalidad en Colombia: Con respecto al impacto de estas campañas y proyectos de cooperación en la reducción de la morbilidad y la mortalidad, aún falta explorar más estadísticas, y aún así, es posible que la información no se logre aclarar totalmente, pues revisando algún material, se encuentra que un buen número de enfermedades se registran como no especificadas o mal definidas, y los renglones de enfermedades endémicas, epidémicas e infecciosas, no aparece desglosado, por lo cual no se saben exactamente los cambios en cuanto enfermedades tratadas por dichas campañas. Además, como lo comentaba el Dr. Héctor Abad Gómez "tenemos pues, que sólo el $38 \%$ de las muertes que ocurren en el país cuentan con un certificado médico y por lo tanto la clasificación total por causas de muerte a penas tiene un valor relativo" (23).

Tomando como base el Informe Currie (1950), el cual presentó por primera vez, de manera organizada las estadísticas relacionadas con la natalidad, la mortalidad, la morbilidad y la situación hospitalaria, se observa ia importante incidencia que tenían enfermedades como las intestinales, la fiebre tifoidea y paratifoidea, la disentería y las enfermedades infecciosas agudas e incluso enfermedades como la lepra (en esta última enfermedad se gastaba un cuarto del total del presupuesto dedicado al Ministerio de Higiene), las cuales no constituían una prioridad en estos programas de cooperación; este hecho, demuestra que no siempre los programas de cooperación en salud responden a las necesidades más sentidas por la población del país que es beneficiario, y que muchos de ellos se realizan más por motivos políticos y económicos coyunturales.

\section{Conclusiones}

El conocimiento y profundización de los programas de cooperación técnica norteamericana en Colombia en el período estudiado, resultan de especial interés para analizar las estrategias de interacción entre estos dos 
gobiernos, pues, a través del campo específico de la cooperación en salud pública, se puede establecer que tales relaciones van más allá del simple ánimo de apoyo a programas sociales o de mejoras en el nivel de vida de la población, para convertirse en verdaderas políticas de Estado que buscan brindar un sistema general de seguridad a sus inversiones en el exterior y promover proyectos políticos como el panamericanismo.

Con lo anterior de ninguna manera se quiere afirmar que los programas de cooperación tuvieron un impacto negativo para Colombia, pues se lograron algunos avances en el control de enfermedades de tipo endémico y epidémico, se capacitó personal en el área de salud pública, y contribuyeron a la reorganización institucional de este sector.

Sin embargo, al tratar de responder la incógnita planteada en el título del artículo, respecto a si las políticas de salud pública en Colombia en el período de 1939 a 1945 se enmarcan dentro de los criterios de mundialización o de desarrollo de local, es necesario destacar cuáles son los aspectos más relevantes de estos dos conceptos:

Por un lado, los procesos de mundialización tienden a unificar modelos, a estimular el comercio y la economía internacionales, a fortalecer vínculos de alianza política y a impulsar planes de apoyo a sectores específicos, de acuerdo con el interés internacional, entre otros. Por otro lado, el desarrollo local busca brindar soluciones particulares a problemas específicos de alcance local o nacional, los cuales no siempre coinciden con las prioridades de los actores internacionales.

Considerando las características típicas de cada una de estas dos posibilidades en las cuales se podría enmarcar las políticas de salud en Colombia en el período estudiado, se puede decir que ellas se desarrollaron con un criterio de mundialización, especialmente por la orientación estratégica de los planes de cooperación dirigidos a fortalecer aspectos económicos, culturales y políticos coyunturales de los Estados Unidos, relacionados con su necesidad de asegurar la alianza de los países latinoamericanos frente al conflicto europeo y posteriormente, con su política de contención del comunismo. Por su parte, la clase dirigente colombiana entró en proceso de negociación de los programas de cooperación, en el cual coincidieron con los intereses norteamericanos, tanto los de sus inversionistas privados, quienes como resultado de la alteración en los flujos comerciales de los países latinoamericanos al antiguo continente vieron fortalecidas sus posibilidades de posesionarse en el mercado de la región, como del mismo gobierno, pues ya los E.U se vislumbraban como la nación hegemónica. EI problema que queda planteado para la evaluación de cualquier programa de cooperación, es el de si éstos responden más a los intereses internacionales que a las necesidades concretas de la población local en la cual son implementados, si se articulan a las políticas de salud que deben ser formuladas a más largo plazo y si los actores locales los asumen acríticamente, aceptándolos pasivamente, solamente porque son una manera de ser tenidos en cuenta en el concierto internacional.

\section{Agradecimientos}

El proyecto se llevó a cabo por una colaboración entre el Laboratorio de Sociedad y Salud del INS y el Centro de Historia de la Medicina "Andrés Soriano Lleras" de la Universidad Nacional de Colombia y fue cofinanciado por la Universidad Nacional, el Instituto Nacional de Salud y Colciencias (Proyecto 2104-04-680-96).

\section{Referencias}

1. Quevedo, E. ¿Políticas de Salud o Políticas Insalubres? De la Higiene a la Salud Pública en Colombia en la primera mitad del siglo XX. Biomédica 1996; 16(4): 345-359.

2. García, M. , Quevedo, E. Uncinariasis y café: los antecedentes de la intervención de la Fundación Rockefeller en Colombia, 1900-1920. Biomédica 1998; 18(1): 5-21.

3. Randall, S. Aliados y Distantes. Historia de las Relaciones Internacionales entre Colombia y Estados Unidos: desde la Independencia hasta la Guerra contra las Drogas. Bogotá: Tercer Mundo Editores. CEl; 1992.

4. Bushnell, D. Eduardo Santos y la Política del Buen Vecino 1938-1942. 2a.ed. Bogotá: El Áncora Editores; 1984. 
5. Sanz de Santamaría, C. Interamericanismo Contemporáneo: Reminiscencia. Bogotá: Academia de Historia de Colombia. Plaza y Janes.

6. Londoño Palacio, A. Memoria del Ministro de Trabajo, Higiene y Previsión Social. Bogotá; 1943.

7. Kranaskas, A. El Servicio Cooperativo Interamericano de salud Pública en Colombia. Bogotá. Ministerio de Salud Pública; 1957.

8. Summer,W. La Política del Buen Vecino. Washington Post. En: Legación de Colombia en Washington. Artículos de prensa. 8 de diciembre; 1943 ..

9. Duroselle, J. B. Política Exterior de los Estados Unidos 1913-1945. México: F.C.E.; 1965.

10. Bejarano, J. "La Economía Colombiana entre 1930 y 1945". En: A. Tirado Mejía. Nueva Historia de Colombia, Vol V. Bogotá: Planeta; 1990.

11. Rodríguez, G. Segunda Administración de López Pumarejo. En: A. Tirado. Nueva Historia de Colombia, Vol I. Bogotá: Planeta; 1990.

12. Thomson, D. Historia Mundial: 1914-1968. 3a.ed. México: F.C.E.; 1980.

13. Ardila, M. (Cambio de Norte? Momentos críticos de la política exterior colombiana. Bogotá: Tercer Mundo Editores e Instituto de Estudios Políticos y Relaciones Internacionales de la Universidad Nacional; 1991.
14. Pecaut, D. Orden y Violencia: Colombia 1930-1954. Bogotá: Siglo XXI Editores y CEREC; 1989.

15. Turbay, G. Conferencias Americanas. Tercera reunión de Ministros de Relaciones Exteriores de las Repúblicas Americanas. Memoria del Ministro de Relaciones Exteriores al Congreso; 1942.

16. Undécima Conferencia Sanitaria Panamericana. Acta Final. Río de Janeiro. Septiembre 6 al 18. Revista de la Facultad de Medicina. 11(12) Junio; 1942.

17. Mac Eachern, M. Comentarios acerca de la Asociación Médica de Hospitales. Revista de Higiene 4(7). Febrero; 1942.

18. Colombia. Ministerio de Salud. Campaña Antipiánica.

19. Colombia. Ministerio de Salud. Campaña Antimalárica.

20. Currie, L. Informe de la Misión del Banco Internacional de Reconstrucción y Fomento; 1950.

21. Bejarano, J. Comentarios Médicos. Revista de la Facultad de Medicina, 10(10): Abril; 1942.

22. Rockefeller Foundation. Annual Report; 1943.

23. Abad Gómez, H. (Cuáles son nuestros mayores problemas sanitarios? Somero análisis sobre las estadísticas de morbilidad y mortalidad en Colombia". Revista de Higiene. 14(1); 1950. 